\title{
DYNAMICS OF ROUGHNESS AND SURFACE REACTIONS AT SOLID ELECTRODES
}

\author{
A. J. Arvia, R. C. Salvarezza* and J. M. Varat \\ * Instituto de Investigaciones Fisicoquimicas Teóricas y Aplicadas (INIFTA), Universidad de La Plata, \\ Sucursal 4, Casilla de Correo 16, (1900) La Plata, Argentina \\ † Departamento de Quimica-Física Aplicada C-Il, Universidad Autónoma de Madrid, (28049) \\ Cantoblanco, Spain
}

(Received 30 January 1992)

\begin{abstract}
Surface disorder and random roughness of electrode surfaces are discussed in terms of Euclidean and fractal models. Roughness development at different metals results from two contributions operating in opposite directions, namely shadowing between growing clusters which leads to rough surfaces, and surface diffusion phenomena which tend to smooth surface irregularities. The second contribution depends on the specific mobility of the deposited metal atoms, the temperature, and the electrolyte composition. Monte Carlo simulations based upon nucleation and growth models show that the growth processes controlled by either surface reactions or mass transport of reacting species, including surface diffusion, produce compact deposits with a weak surface disorder. Conversely, the growth process under mass transport control involving a negligible surface diffusion generates open structures with a strong surface disorder. Rough metal deposits exhibiting a fractal behaviour are considered. The fractal dimension provides a quantitative description of the degree of disorder, the growth mechanism and the reactivity of those surfaces in relation to electrocatalysis.
\end{abstract}

Key words: fractals, electrode roughness, Monte Carlo simulations, growth modes, surface dynamics.

\section{INTRODUCTION}

Electrocatalytic reactions at solid electrodes involve the participation of adsorbed species acting either as a short half life intermediate or as a poison depending on the electrochemical system. The presence of different adsorbed species at solid electrodes opens the possibility of coadsorbate formation, and rearrangement of the substrate surface atoms. The contributions of these processes, namely the proper electrocatalytic reaction, the formation of both poison and coadsorbate species, and the electrode surface rearrangement, determine the lifetime of the electrode material. Likewise, the efficiency of the solid electrode for a particular reaction depends on the number of active sites per unit area, and the surface-to-volume ratio, which are directly related to the optimum reaction rate and the greatest selectivity, respectively.

Electrochemists were concerned about these two important aspects of electrochemical kinetics since the pioneering investigations on the hydrogen evolution reaction (HER) on smooth and rough $\mathbf{P t}$ electrodes[1] in aqueous solution under quasistationary conditions. However, only in recent years could an explanation of the specific reactivity of the Pt electrode in terms of the metal surface be advanced[2-4] because of the progress made on the structure of solid surfaces, and the possibility of obtaining electrochemical data complemented by in situ surface analysis and scanning tunnelling microscopy (STM) at the atomic level[5] for extremely clean and well-defined single crystal electrochemical systems. These advances have shortened the gap existing between heterogeneous catalysis and electrocatalysis [6], as they made it possible to know the type and the density of intrinsic and extrinsic defects at solid electrode surfaces, and to follow more precisely the dynamic behaviour of several adsorbates of electrocatalytic interest.

In contrast to single crystal electrochemistry, the rationale of the surface-to-volume ratio at solid electrodes is very limited because knowledge of rough solid surfaces at the atomic level is still rather poor. There is neither a single accepted criterium for defining both the surface smoothness and the surface roughness nor a general structural theory applicable to rough solid surfaces, except some crude models. However, recent work on the matter[9] allows us to approach a more comprehensive concept of roughness which is applicable to electrochemical systems.

This work offers a survey of recent advances in the development of different kinds of solid metal surfaces, and their characterization and specific properties, including reactivity characteristics. This information constitutes part of the basic physicochemical framework for the design of real solid electrode surfaces of interest for electrocatalysis.

\section{SOLID ELECTRODE SURFACES}

\subsection{Single crystal metal surfaces}

The surface structure of single crystals undergoes several changes such as relaxation, reconstruction and relaxation-reconstruction induced by changes of the surface composition. These modifications which can be detected by low energy electron diffraction (LEED) and surface crystallography[10], generate a certain roughness inherent to the solid surface, so 
that the real atomic smoothness implying atoms at identical equilibrium positions in a well-ordered array, can only be found in small domains (Fig. 1)[11]. The crystallographies of the small flat domains are generally defined by low Miller indices, whereas the surface irregularities such as steps, corners, and kinks lead to a periodic lattice described through higher Miller indices.

Due to relaxation, surface atoms in single crystals are accommodate in new equilibrium positions that change $\Delta d_{n, n+1}$, the interlayer distance in the bulk with respect to $\Delta d_{1,2}$, the distance between the first and the second layer of atoms or ions. This rearrangement of surface atoms decreases the free energy of the system to a minimum value, causing the appearance of roughness. In this case, changes in Miller indices of surface atoms can be ascribed to changes in the atom density per unit area, the latter being proportional to the reciprocal of the corresponding lattice constant. Accordingly, the roughness of the single crystal surface can be defined with respect to the most densely packed metal(111) surface as the $\Delta d_{1,2} / \Delta d_{n, n+1}$ percentage ratio for the different crystallographic faces of face-centred cubic (fcc) and body-centred cubic (bcc) lattices (Fig. 2)[12]. As examples seen in this figure, the fcc (411) and the bcc (410) faces imply roughness values equal to 5.5 and 7, respectively.

Results obtained for the $\mathrm{H}$ atom electrosorption processes on Pt single crystal electrodes characterized by LEED and other surface techniques under ultra-high-vacuum (UHV) conditions $[7,8]$ provided conclusive information about the influence of the crystalline structure on that reaction. The corresponding voltammograms run in acid solution exhibit a number of conjugated peaks which become sensitive to the crystallographic structure of the $\mathrm{Pt}$ single crystal surface under constant composition, temperature and applied potential conditions [13] (Fig. 3). On Pt single crystals in acid solution the locations of the conjugated $\mathrm{H}$ atom electrosorption current peaks change according to the Miller indices

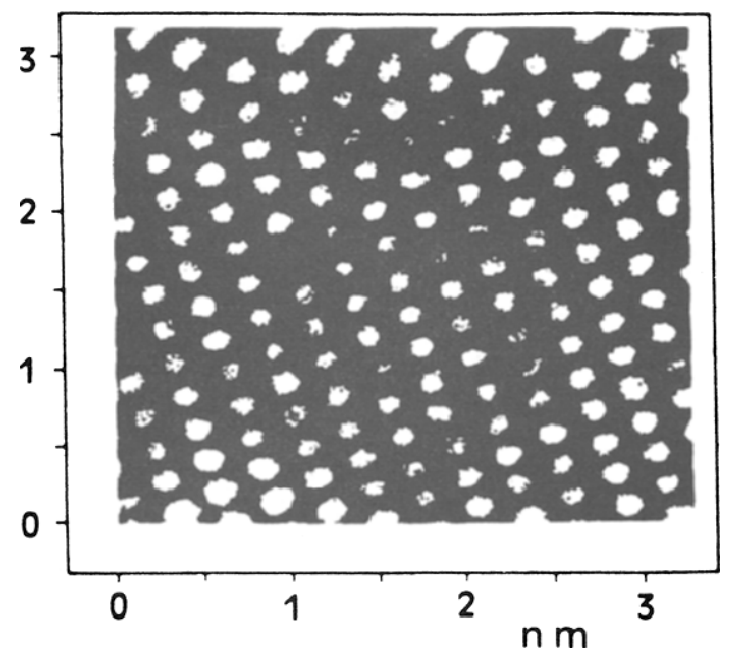

Fig. 1. Atomic resolution STM image of $\mathrm{Al}(111)$ $(3.4 \times 3.4 \mathrm{~nm})$ top view, corrugation: $0.03 \mathrm{~nm}$ (reproduced with permission from Ref. [11]).

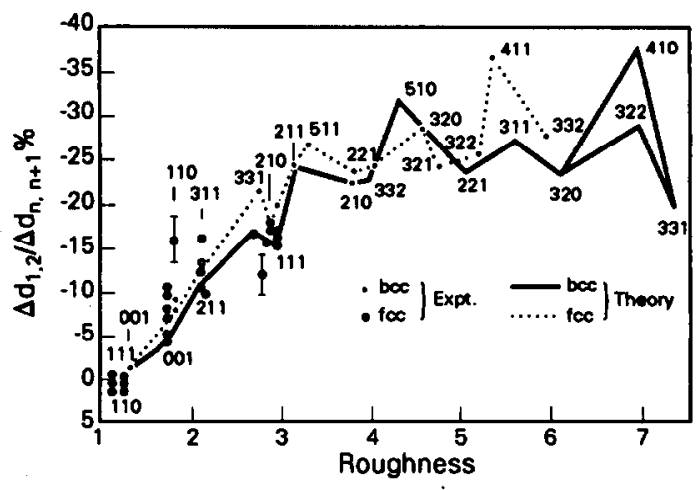

Fig. 2. Experimental and theoretical first layer relaxation (in per cent) as a function of roughness for several boc and fcc surfaces (reproduced with permission from Ref. [12]).

in apparent agreement with the surface energy distribution of the different stepped Pt surfaces[13]. Analogous results have been reported for the $O$ atom electrosorption[14], the underpotential deposition (upd) of foreign atoms $[15,16]$ and the electrooxidation of relatively simple organic molecules[17-19] on several single crystal metal electrodes.

Nevertheless, from a strict crystallographic standpoint single crystal surfaces at $T \gg 0 \mathrm{~K}$ involve a certain concentration of surface defects which is usually unknown. This fact, in addition to changes promoted at the electrode surface by the electrochemical reaction itself and the influence of the electrolyte composition, makes the interpretation of voltammetric results somewhat difficult $[20,21]$. As recently reported[22], the topography of $\mathrm{Au}$ single crystal electrodes in different aqueous electrolytes, followed by in situ STM images, can be considerably modified by adsorption-desorption cycles. These surfaces at large length scales revealed a strong disorder and an increase of roughness. For Au(111)

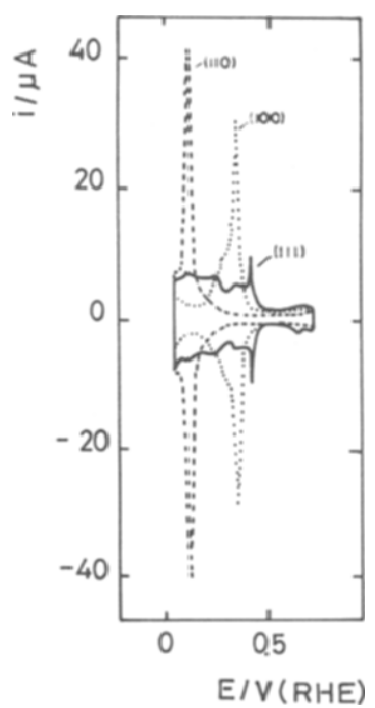

Fig. 3. H-Adatom electrosorption voltammograms for $\mathrm{Pt}(111), \mathrm{Pt}(100)$ and $\mathrm{Pt}(110$ in acid solution (reproduced with permission from Ref. [13]). 
electrodes after either a $\mathrm{Cl}^{-}$ion or an $\mathrm{O}$ atom adsorption-desorption cycle, the topography of the surface is disturbed up to a distance of about $10 \mathrm{~nm}$ around the adsorption site[23]. Comparable surface changes have been also reported for a number of foreign metal upd on several substrates[24, 25$]$.

\subsection{Faceting and electrofaceting}

The relaxation of surface atoms produces changes in the equilibrium position of atomic bonds causing a strong local compression which provokes the reconstruction of the outermost atomic layers by changing only the bond angles, leaving the coordination number and the rotational symmetry of surface atoms unaltered[5]. Otherwise, the surface atom modification described as surface reconstruction implies simultaneous changes in bond angles, cordination number and rotational symmetry of surface atoms. These changes tend to generate more stable and longer lasting surface structures. In general, for any crystallographic array of surface atoms the electronic properties change in going from step to terrace atoms. The average work function decreases as the step density increases and as the number of nearest neighbour surface atoms decreases. This means that inner relaxation processes are favoured at both step and kink sites[5]. From the surface electrochemistry standpoint, these defects can be considered within the group of the most reactive sites.

The surfaces of polycrystalline (pc) materials commonly used in heterogeneous processes are poorly defined. However, this situation can be improved through surface atom rearrangements at those materials which modify the distribution of crystallographic faces, and consequently the reactivity of the solid surfaces. The number of metal surfaces of electrochemical interest can be accommodated through faceting techniques leading to reproducible, preferred crystallographic orientations.

Faceting can be described as a surface atom rearrangement yielding a surface with a valley-hill topography and flat domains[10]. Faceting of metal surfaces can be produced either thermally, under high energy particle impact, chemically (etching), or electrochemically (electrochemical faceting). The electrochemical faceting of metals[26] is based on the distinct electrochemical reactivity of surface atoms with different Miller indices. Cyclic processes involving metal electrodissolution/metal electrodeposition can alter drastically the crystallographic characteristics of the solid electrode surface $[27,28]$. After the electrochemical faceting the behaviour of the system approaches that of reconstructed single crystal electrode surfaces in the same environment[28]. Definite mono-orientation effects for a number of pc fcc metals can be accomplished through the proper adjustment of the potential routine, the electrolyte composition and the temperature[27].

Electrochemical faceting was also applied to polyfaceted single crystal metal electrodes yielding surface patterns resembling very closely those ones expected from faceting of ideal single crystal spheres[26].

\section{SURFACE DISORDER}

STM imaging at the atomic level shows that welldefined planes exist only for very restricted surface domains. On the other hand, real surfaces of solid materials exhibit strong disorder so that a quantitative description of their topography becomes extremely difficult. Euclidean models have been used to represent rough surfaces, although for very irregular systems the concepts of fractal geometry are more suitable for this purpose[29].

Fractals are disordered systems, the degree of disorder being expressed in terms of a non-integral dimension, $D_{\mathrm{H}}$, which satisfies the condition $D_{\mathrm{H}}<d$, where $d$ stands for the dimension of the space in which the object is embedded[29]. Fractal geometry treats disorder as an intrinsic rather than a perturbative phenomenon and it has been extensively applied to understand geometric, physical and chemical properties of complex systems.

The type of disorder formed on solid surfaces can be schematically illustrated on the basis of continuum models[9] (Fig. 4). The distinction of the models can be made through the invariance of certain properties. Thus, the perfectly ordered surface (Fig. 4a) is dilation and translation invariant. The weakly disordered surface which can be used as a model for stepped surfaces (Fig. 4b) comprises only local dilation and translation invariance, and the singularities present on the surface can be considered as a sub-fractal set. The anisotropic strong disordered surface denoted as a self-affine fractal (Fig. 4c) implies dilation invariance and only microscopic translation invariance. This example lies in the borderline between weakly and strongly disordered surfaces. Finally, the isotropic strong disorder which characterizes a self-similar fractal involves a dilation

a

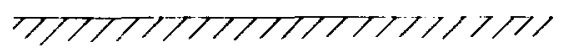

b

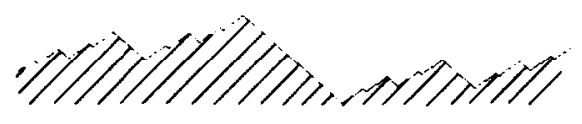

C

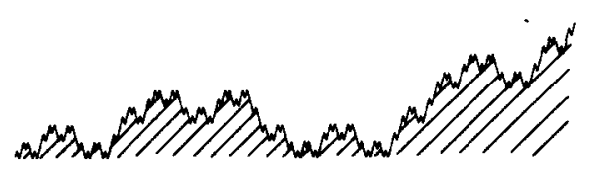

d

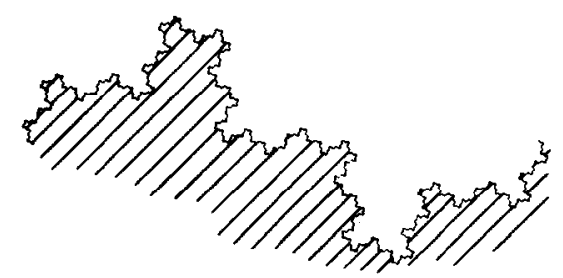

Fig. 4. Different types of disorder (continuum models). (a) No disorder; (b) weak disorder; (c) anisotropic strong disorder (self-afiine); (d) isotropic strong disorder (self-similar) (reproduced with permission from Ref. [9]). 
invariance and no translation invariance (Fig. 4d) [9].

The concept of surface disorder brings up the meaning of real surface area measurements of porous electrode materials by using a certain foreign molccule as a yardstick. Results from this type of measurement do not represent the real area of the solid surface, unless the size of the probe molecule is smaller than the size of the smallest irregularities at the rough surface (Fig. 5)[30]. Hence, only for the latter case does the resulting quantity represent the actual area of the rough surface. The relative error due to the influence of excluded volumes depends on both the size of the yardstick and the geometry of the surface. The influence of the latter as well as the characteristics of the surface geometry are considered in detail later. Likewise, for a recent review about surface area measurements applied to electrochemistry the reader can refer to Ref. [31].

\subsection{Surface area measurements}

Voltammetry is perhaps one of the simplest in situ methods which has been proposed for the evaluation of the real surface area of solid electrodes, being applicable at any instant during the lifetime of the electrode. This method is particularly suitable for a metal electrode at which a monolayer of a welldefined adsorbate can be formed and $q_{m}$, the adsorbate monolayer charge density value, can be checked by using an independent method. $Q_{\mathrm{d}}$, the voltammetric charge involved in the test electroadsorption reaction, can be obtained by voltammetry. Then $A$, the real surface area of the electrode, is obtained from the ratio:

$$
A=Q_{\mathrm{d}} / q_{\mathrm{m}}
$$

and $R$, the roughness factor is defined as:

$$
R=A / A_{s},
$$

$A$, being the geometric area (apparent area) of the solid electrode.

Although the use of voltammetry for surface area measurements involve certain limitations [31], it can be employed for several metals, such as $P t$ and $R h$, by considering the $\mathrm{H}$ atom electrosorption; for $\mathrm{Pt}$, $\mathrm{Rh}$, and $\mathrm{Au}$ taking into account the $\mathrm{O}$ atom electrodesorption; and for $\mathrm{Ag}$ and $\mathrm{Cu}$ electrodeposits by watching the upd of either $\mathrm{Pb}$ or $\mathrm{Cd}$ as test reactions[32]. In all these cases, acceptable values for the real surface area and the roughness factor can be obtained because the size of the yardsticks is less

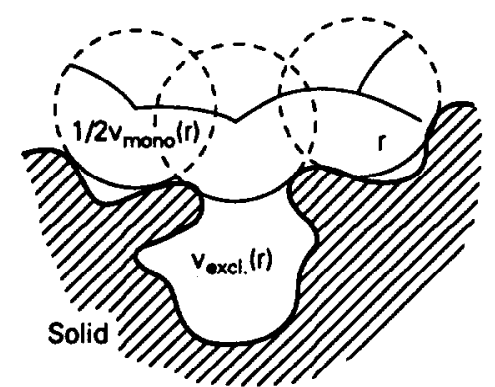

Fig. 5. Scheme of a monolayer of molecules of radius $r$ coating a corrugated surface (reproduced with permission from Ref. [30]). than the size of the smallest irregularities at the metal surfaces.

\subsection{Roughness development at metal surfaces}

It is important to consider the variables controlling roughness development at solid surfaces because of the role played by the electrode roughness in electrochemical kinetics. It is known that irreversible growth phenomena often result in rough surfaces, so that this type of process becomes particularly attractive for the present purpose.

The following three growth procedures have been investigated to develop rough metal surfaces and to determine the corresponding critical variables controlling roughness development.

(i) The in situ formation of a highly dispersed metal overlayer from the electroreduction of an anodically formed metal oxide layer [33, 34].

(ii) The electrodeposition of soluble metal ions from aqueous solution. This type of reaction has been extensively studied in relation to the electrocrystallization of metals [35].

(iii) The metal deposition from the vapour phase.

The characteristics of the metal deposits obtained through these procedures depend on the working conditions, principally on the nature of the substrate, the overlayer growth rate, the temperature, and the electrolyte composition. Recent works on the kinetics and growth mode of metal electrodeposits[36-38] have shown that the influence of those variables can be explained by considering the roughness development as the result of competition between growing clusters, leading to shadowing and accordingly to roughness development, and surface diffusion of depositing particles, tending to smooth down the irregularities formed during growth. Shadowing is enhanced and the effect of surface diffusion is decreased at high overlayer growth rates. Typical rates to develop rough patterns are within the range $15-150 \mathrm{~nm} \mathrm{~s}^{-1}$ for $T<0.3 T_{\mathrm{m}}$, where $T_{\mathrm{m}}$ is the melting temperature range[38]. In these cases surface diffusion becomes a slow process for smoothing surface irregularities.

The kinetics of the early stages of Au electrodeposition on $\mathrm{Au}(\mathrm{pc})$ from a $\mathrm{Au}$ oxide layer can be explained through a nucleation and growth mechanism[36]. The structure generated by nuclei growth in the long-term range depends on the proper growth rate. Thus, rough Au electrodeposits can be grown at $100 \mathrm{~nm} \mathrm{~s}^{-1}$ by applying a relatively large cathodic overpotential. In this case the value of $R$ can be estimated through Equations (1) and (2) by taking $0.42 \mathrm{mC} \mathrm{cm}^{-2}$ as the charge density of the $O$ atom monolayer on $\mathrm{Au}(\mathrm{pc})$.

For $\mathrm{Au}$ and $\mathrm{Pt}$ rough surfaces, the value of $R$ is proportional to $q$, the electrodeposited charge per substrate area unit (Fig. 6). For low growth rates $\left(0.1 \mathrm{~nm} \mathrm{~s}^{-1}\right)$ and low cathodic overpotentials, a smooth metal surface with a value of $R$ practically independent of $q$ can be obtained (Fig. 6) [39]. For a constant $q$, the value of $R$ fulfils a $1 / 2$ th power dependence on $j_{M}$, the maximum electrodeposition apparent current density at constant potential (Fig. 7)[39]. Similar linear $R$ vs. $q$ plots can be obtained for Ag electrodeposits grown at high cathodic overpotentials in aqueous solution[40]. 


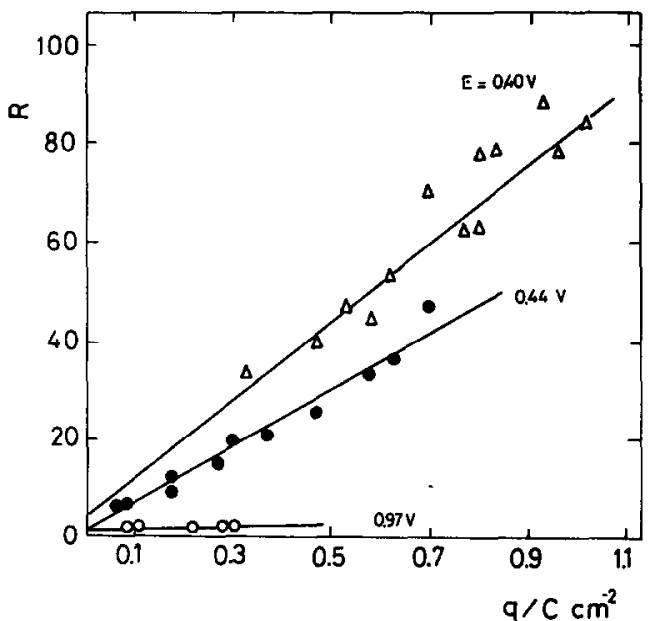

Fig. 6. $R$ vs. $q$ plots for metal electrodeposition from the electroreduction of metal oxides at different potentials, $E$. ( $\triangle$ ) Pt $(E=0.40 \mathrm{~V})$; (O) Au $(E=0.44 \mathrm{~V})$; (O) Au $(E=0.97 \mathrm{~V}$ ) (reproduced with permission from Ref. [39]).

The $R$ vs. $q$ dependences resulting for electrochemically grown deposits can be compared with $R$ vs. $h$, the average layer thickness dependences for vapour phase Au deposits grown at the same rate $\left(100 \mathrm{~nm} \mathrm{~s}^{-1}\right)$ and substrate temperature $(298 \mathrm{~K})$. For vapour deposited Au layers the value of $h$ becomes proportional to the deposited mass $(m)$. The latter is equivalent to the value of $q$ for $A u$ electrodeposits. For Au films grown from the vapour phase the value of $R$ increases with $h$ up to $h=120 \mathrm{~nm}$, but for $h>120 \mathrm{~nm}$, it remains practically constant (Fig. 8) [41], otherwise, for electrodeposited $\mathrm{Au}$ the value of $R$ increases near linearly with $q$. These results indicate that $\mathrm{Au}$ deposits grown from the vapour phase are more compact than those obtained from $\mathrm{Au}$ electrodeposition. For the latter, voids and channels extend deeply in the rough $\mathbf{A u}$ deposit, leading to an open structure.

\subsection{Euclidean geometric models for rough metal surfaces}

Rough metal surfaces can be described as heterogeneous systems made of metal and void domains.

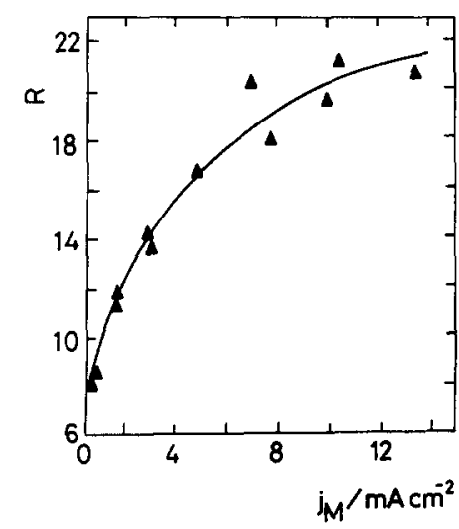

Fig. 7. $R$ vs. $J_{M}$ plot for Au electrodeposition from the electroreduction of Au oxide layers (reproduced with permission from Ref. [39]).

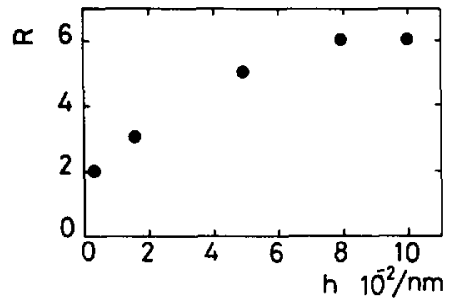

Fig. 8. $R$ vs. $h$ plot for vapour deposited Au layers. $h$ was measured by using a profilometer.

The relative distribution and geometry of these domains can be associated with two limiting situations, one concerning Euclidean surfaces, and another related to fractal surfaces. In the former case, the metal/void ratio can be either a function of $h$ as in conical-type growth patterns, or $h$ independent as in cylindrical and square cluster growth patterns. These two simplified approaches were used for describing the complex ellipsometric parameters resulting for rough metal electrode surfaces[42].

STM-SEM images of Au electrodeposits grown at a low rate reveal large rounded grains, the resulting structure being described as an array of hemispherical elements. For this geometric model $R$ becomes independent of both grain size and $q$ values, with $R \cong 2$ (Fig. 6) [37].

STM images of Au and Pt deposits grown at high rates and at $298 \mathrm{~K}$ reveal small rounded grains (on the order of a few nanometres) with intergranular voids (see Fig. 1 in Ref. [43]). SEM micrographs of the deposit cross-section also show that the grains are elongated, forming a columnar-like structure[44]. Therefore, the simplest structural model for the deposits consists of columns of average radius $r$ and average height $h$, the value of $h$ being approximately equal to the metal overlayer thickness (Fig. 9). According to this model, for $h \gg r$ the value of $\boldsymbol{R}$ can be obtained from the ratio[37]:

$$
R \simeq \pi h / 3 r .
$$

Equation (3) allows $r$ to be estimated from the voltammetrically determined value of $R$. The value of $h$ can be calculated from the SEM micrographs of the electrodeposit cross-section [44]. The values of $r$ evaluated from Equation (3) are in reasonable agreement with those obtained from STM imaging[37, 44]. Moreover, by considering that $h \propto q$, the nearly

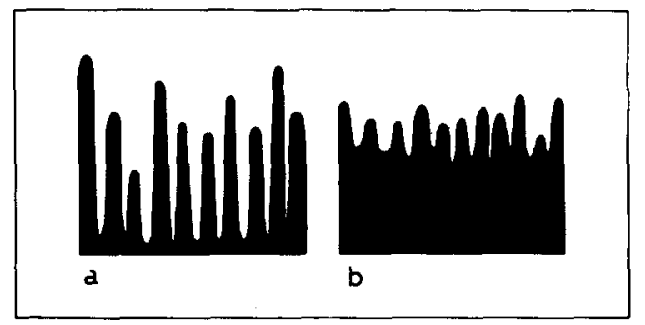

Fig. 9. 2-D profiles of the columnar models used for the structures of metal deposits. (a) Deposit grown from the electroreduction of metal oxides; (b) vapour deposited metal. 
linear $\boldsymbol{R}$ vs. $q$ plots observed for metals grown far from equilibrium can be justified (Fig. 6).

On the other hand, for Au layers grown from the vapour phase Equation (3) can be rewritten in terms of $\zeta$, the average interface thickness, instead of $h$. The value of $\zeta$ can be estimated from the maximum difference in the height of columns as determined from the STM images covering the scan length $L$. For $L \gg h$, the dependence of $\zeta$ on $h$ (Fig. 10) is given by[41]:

$$
\zeta \propto h^{\beta}
$$

with $\beta \cong 0.3$. This figure suggests that for these $A u$ overlayers only a fraction of the entire Au layer thickness contributes to the roughness of the deposit as discussed in the preceding section.

In Equation (3) an $h$-independent value of $r$ has been assumed. However, recent data[45, 46] have shown that $r$ actually depends on $h$ according to:

$$
r \propto h^{p}
$$

and the value of $p$ depends on the overlayer preparation conditions and it can be as small as $0.2[46]$. This means that the properties of the rough metal surfaces become more complex than those predicted by the simple geometric models. This leads to new approaches for describing the rough metal surfaces in terms of the fractal geometry[29] which are discussed in Section 6. Nevertheless, the simple columnar model allowed a first quantitative analysis of the roughness decay caused by surface mobility of deposited metal atoms. The relative contribution of

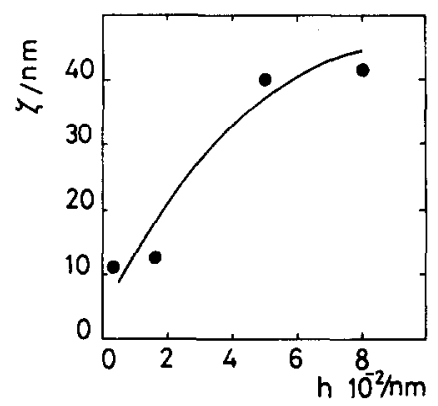

Fig. 10. $\zeta$ vs. $h$ plot for vapour deposited Au film. The values of $\zeta$ were measured as the maximum height difference in $3000 \times 3000 \mathrm{~nm}$ STM images. roughness decay depends on the ratio between $T$, the temperature of the rough metal and $T_{m}$, its melting temperature[47]. Roughness decay appears during the formation of the rough metal overlayer as well as a post-deposition roughness changes operating either under open circuit[44] or applied potential conditions $[48]$.

\subsection{Post-deposition effects at rough metal surfaces}

The spontaneous decrease in the value of $R$ which can be easily followed through conventional electrochemical experiments (Fig. 11)[44] belongs to a general type of surface relaxation phenomena. Roughness decay at metal overlayers occurs at any ambient condition [49].

For columnar $\mathrm{Au}$ and $\mathrm{Pt}$ electrodeposits in contact with the electrolyte solution, the kinetics of roughness relaxation fits linear $R$ vs. $t^{-1 / 4}$ relationships [44] which are similar to those describing the evolution of particle size in coalescence phenomena[49]. For the latter $r$, the average particle radius increases with time $(t)$ according to the following equation:

$$
\left(r-r_{0}\right)^{4}=2 \gamma a^{4} D_{i} t / k T,
$$

where $\gamma$ is the surface tension, $a$ is the metal lattice parameter, $D_{i}$ is the surface diffusion coefficient of metal atoms, and $r_{0}$ is the initial average radius of the particles forming the electrodeposit. Using the simple columnar model (Fig. 9) and further assuming that $\mathrm{d} h / \mathrm{d} t \ll \mathrm{d} r / \mathrm{d} t$, Equations (3) and (6) lead to:

$$
R=h /\left(2 \gamma a^{4} D_{i} t / k T+r_{0}\right)^{1 / 4} .
$$

$D_{i}$ can be obtained from Equation (7), for various metals immersed in different electrolytes. Thus, for rough $\mathrm{Au}$ and $\mathrm{Pt}$ specimens kept in contact with $1 \mathrm{M}$ $\mathrm{H}_{2} \mathrm{SO}_{4}$ under open circuit one obtains, $D_{1}=5$ $\times 10^{-14} \mathrm{~cm}^{2} \mathrm{~s}^{-1}$ for $\mathrm{Au}$, and $D_{1}=10^{-18} \mathrm{~cm}^{2} \mathrm{~s}^{-1}$ for Pt. The temperature dependence of $D_{i}$ obeys an Arrhenius plot in the $273-325 \mathrm{~K}$ range yielding $\Delta Q^{*}$, the activation energy for surface diffusion; $\Delta Q^{*}=$ $14 \mathrm{kcal} \mathrm{mol}^{-1}$ for $\mathrm{Au}$, and $\Delta Q^{*}=19 \mathrm{kcal} \mathrm{mol}^{-1}$ for Pt[44].

The mechanism of surface diffusion as described for metal/gas interfaces, comprises two limiting rate determining steps depending on whether metal atoms or surface vacancies become the slowest moving particles[47]. When the diffusion of metal
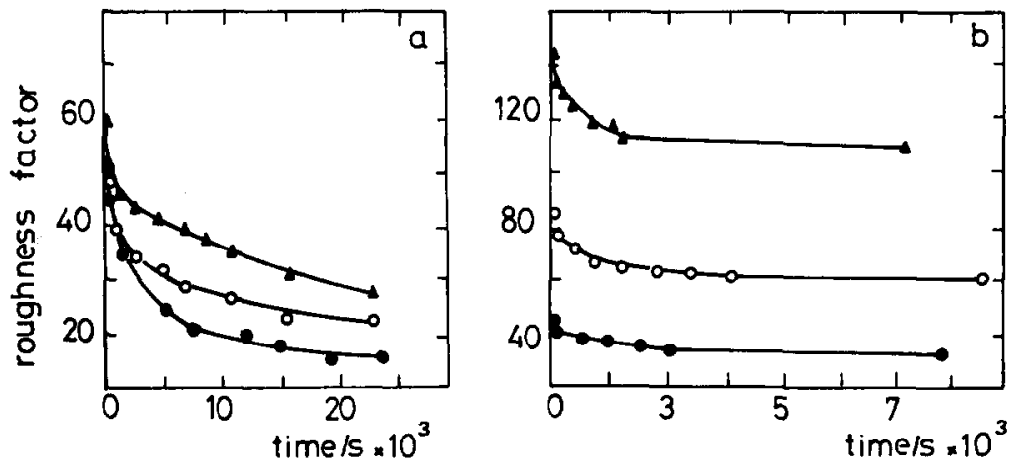

Fig. 11. $R$ vs. $t$ plots for metal deposits grown from the electroreduction of metal oxide layers. (a) Au deposits produced from a constant amount of Au oxide. Temperature influence; (b) Pt deposits obtained from different amounts of Pt oxide, $T=298$ (reproduced with permission from Ref. [44]). 


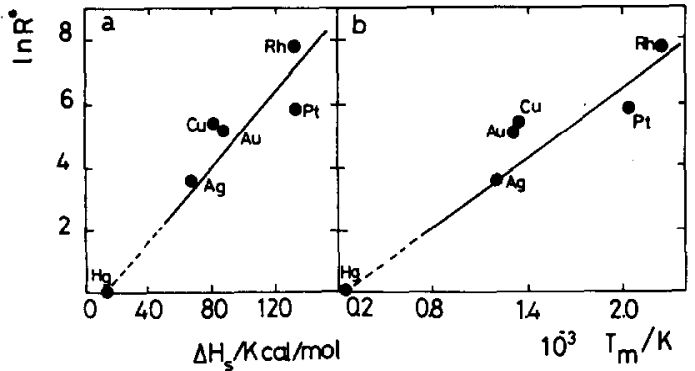

Fig. 12. (a) $\ln R$ vs. $\Delta H_{\mathrm{s}}$ and (b) $\ln R$ vs. $T_{M}$ plots for different metal electrodeposits (reproduced with permission from Ref. [51]).

atoms is rate determining, $\Delta Q^{*}=0.54 \Delta H_{3}$, where $\Delta H_{3}$ denotes the enthalpy of sublimation of the metal. This situation is approached when $T / T_{\mathrm{m}}>$ 0.75 . Conversely, when $T / T_{\mathrm{m}}<0.75$, the surface diffusion of vacancies becomes rate determining yielding $\Delta Q^{*}=0.24 \Delta H_{3}$. A direct comparison between the $\Delta Q^{*}$ values, predicted by the different rate determining steps, and the experimental values derived for rough $\mathrm{Au}$ and $\mathrm{Pt}$ electrode surfaces, indicates that the roughness decay for these metals can be explained as a surface diffusion mechanism involving vacancies.

It should be noted that the value of $D_{i}$ depends on both the applied potential and the presence of adsorbable species in the solution. For instance, a small amount of pyridine added to $1 \mathrm{M} \mathrm{H}_{2} \mathrm{SO}_{4}$ produces a strongly bound adsorbate on $\mathrm{Au}$ modifying the rate of roughness relaxation by decreasing the value of $D_{1}$ by about one order of magnitude[50]. Otherwise, the addition of $\mathrm{Cl}^{-}$ions to the electrolyte solution produces the reverse effect due to the formation of a complex-type adsorbate on $\mathrm{Au}$, the latter being a precursor for $\mathrm{Au}$ electrodissolution in that medium.

\subsection{The surface mobility of metals and the development of surface roughness}

Let us consider the influence of the nature of the electrodepositing metal on the development of the rough overlayer. Earlier experimental results obtained for the potentiostatic growth of rough $\mathbf{R h}$, $\mathrm{Pt}, \mathrm{Au}, \mathrm{Cu}$, and $\mathrm{Ag}$ deposits have shown a linear increase of $R$ with $q$, the electrodeposited metal charge[37, 39, 40, 51] (Fig. 6); then, the value of $R^{*}$, the slope of the $R$ vs. $q$ linear relationship, is given by:

$$
R^{*}=z F\left(\Delta R / \Delta q_{\mathrm{d}}\right)
$$

$R^{*}$ defines the roughness of the deposit per mole of electrodeposited metal ion referred to the substrate area. It was found that the value of $R^{*}$ increases in the order $\mathbf{A g}<\mathbf{A u}<\mathbf{C u}<\mathbf{P t}<\mathbf{R h}[51]$.

On the other hand, the experimental values of $R^{*}$ can be related to $\Delta H_{\mathrm{s}}$ and $T_{\mathrm{m}}$ according to the equation:

$$
R^{*}=K \exp \left(k_{1} \Delta H_{\mathrm{a}}\right)=K \exp \left(k_{2} T_{\mathrm{m}}\right)
$$

where $K$ is a constant to be discussed further on. Equation (9) offers the possibility of considering other aspects of the surface metal atom diffusion mechanism.
Let us consider that $R^{*}$ is proportional to the reciprocal of $D_{i}$. The latter can be expressed through the already known equation[52]:

$$
D_{1}=K^{\prime} \exp \left(\Delta S^{*} / R\right) \exp \left(-\Delta H^{*} / R T\right),
$$

where $\Delta S^{*}$ and $\Delta H^{*}$ stand for the entropy and the enthalpy of activation for the surface diffusion process, respectively, and $K^{\prime}$ is a pre-exponential factor comprising a number of parameters resulting from the rate process theories[52].

The experimental activation energies derived for surface diffusion of $\mathrm{Au}$ and $\mathrm{Pt}$ atoms are close to $0.2 \Delta H_{\text {. }}$. Thus, Equation (10) can be written as:

$$
D_{i}=D_{0} \exp \left(-0.2 \Delta H_{v} / R T\right),
$$

where $D_{0}=K^{\prime} \exp \left(\Delta S^{*} / R\right)$. Combining Equations (9) and (11), linear $\ln R^{*}$ vs. $\Delta H$, and $\ln R^{*}$ vs. $T_{\mathrm{m}}$ relationships are obtained, in agreement with experimental results (Fig. 12). Hence Equation (10), which is usually employed as an empirical equation[47], can be justified through a surface diffusion mechanism for moving surface atoms. Then, as the validity of Equation (9) has been proved for a number of metals, it becomes useful for predicting the magnitude of $\boldsymbol{R}$ for metal overiayers prepared under fast growing operating conditions. This is certainly an interesting achievement regarding the design of large surface area metal electrodes.

\section{ELECTROCHEMICAL REACTIONS ON ROUGH ELECTRODES}

A number of electrochemical reactions have already been investigated by employing reproducible and relatively well-defined rough metal electrode surfaces of $\mathrm{Ag}, \mathrm{Au}, \mathrm{Cu}, \mathrm{Pt}$ and $\mathrm{Rh}$.

From the standpoint of electrochemical kinetics, a rough metal surface actually behaves as a nonhomogeneous material involving metal domains which behave as small metal clusters, and other regions with different local reactivity. The reactivity sequence of these domains is qualitatively similar to the reactivity order of single crystal cluster surfaces $[53,54]$.

\subsection{Surface reactions}

Electrodispersed Pt electrodes were applied for the first time to investigate several surface electrooxidation reactions such as the electro-oxidation of formaldehyde, methanol[56], adsorbed CO[56], reduced $\mathrm{CO}_{2}[57]$ and other organic fuels[58] which are directly related to electrochemical energy conversion. In these cases a considerable enhancement of the electrocatalytic reaction rates was found. This fact is due to the increase in the active surface area and to the decrease of the anodic polarization for the electro-oxidation processes. In these cases, the surface concentration of electrocatalytic poisons is considerably diminished as concluded from the electromodulated ir spectra of electrodispersed Pt electrode run in $\mathrm{CO}$ saturated acid electrolytes[58]. Similar results have been reported for the electrooxidation of methanol[59].

On the other hand, the particular characteristics of electrodispersed $\mathrm{Pt}$ electrodes can also be related to 
the relatively small size of the metallic domains. Accordingly, specific size effects, similar to those described for dispersed catalysts, should appear at electrodispersed Pt electrodes[60]. Furthermore, these electrodes should also refiect the influence of faceting which accompanies the development of the rough metal surface on the kinetics of the electrochemical reactions.

\section{2. upd and opd metal electrodeposition on rough substrates}

The study of reactions such as $\mathrm{Ag}$ and $\mathrm{Cu}$ upd on rough $\mathrm{Pt}$ electrodes allows the enhancement of the contribution of processes mainly dominated by surface atom diffusion such as those related to spillover effects. These contributions are practically overlooked when metal upd reactions proceed at smooth electrode surfaces.

The electrodeposition of $\mathbf{A g}$ and $\mathrm{Cu}$ from diluted metal-ion-containing solutions on electrodispersed $\mathrm{Pt}$ as followed through conventional voltammetry, is controlled by the diffusion of metal ions from the bulk of the solution towards the tips of the columns forming the rough metal structure[61]. In this case, each tip behaves as an ion-collecting site during the electrodeposition reaction. This effect depends on the roughness factor value, the potential scan rate, and the metal ion concentration in the solution.

Perhaps one of the most interesting features related to the early stages of metal electrodeposition on rough metal substrates is the inhibition of bulk metal electrodeposition either partially or completely[61]. In this case, the 2-D metal overlayer growth is favoured because of the depositing metal adatom diffusion over the 2-D growing submonolayer domains, towards free substrate sites at the wall of the voids (Fig. 13). The probability of surface adatom diffusion increases as the metalmetal bond becomes weaker than the metalsubstrate bond. The high mobilities of depositing $\mathrm{Cu}$ and $\mathrm{Ag}$ adatoms on either $\mathrm{Cu}$ or $\mathrm{Ag}$ covered $\mathrm{Pt}$ surface domains agree with the poor capability of these metals for roughness development at room temperature, as can be seen through the correspond-

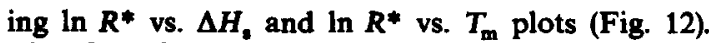
Therefore, for fractional coverages of the substrate by the 2-D metal layer, the surface diffusion process results in the inhibition of the bulk metal formation. These results correlate to the $2-\mathrm{D} \Rightarrow 3-\mathrm{D}$ reversible rearrangement of surface atoms taking place at the monolayer level during the electrodeposition of metals[24].

\section{A MODEL FOR ROUGHNESS DEVELOPMENT. MONTE CARLO SIMULATIONS}

Monte Carlo calculations of growing metal overlayers were made on a 2-D square lattice substrate based upon nucleation and growth model. The calculations have taken into account the following contributions [39]:

(i) The particle diffusion from either the bulk of the solution or the oxide phase to the 2-D flat substrate. The latter represents a cross-section of the ideal 3-D substrate.

(ii) The particle sticking probability, $P_{\mathrm{a}}$, to form nuclei is calculated as the product of a potential dependent term, $\boldsymbol{P}_{n}$, and another term, $\boldsymbol{P}_{s}$, which depends on the number of neighbours to the sticking site.

(iii) The growth of nuclei through the sticking of arriving particles.

(iv) The surface diffusion of sticking particles up to a maximum distance, $l_{*}$, for final attachment to a surface site involving the largest coordination number.

By changing $P_{\eta}$, the model allows the simulation of the growth processes under different kinetic controls. Thus, for $P_{\eta} \Rightarrow 0$ the growth process is controlled by a surface reaction, whereas for $P_{\eta} \Rightarrow 1$ it becomes mass transport controlled. Snapshots obtained for a number of deposited particles, $n=1000$, a number of atoms in the critical nucleus, $n^{*}=2, l_{n}=3$, and different $P_{\eta}$ values are shown in Fig. $14 a-c$. The value of $R$, the roughness of the 2-D deposit, is defined as the ratio between the number of particles in the growing 2-D profile and the
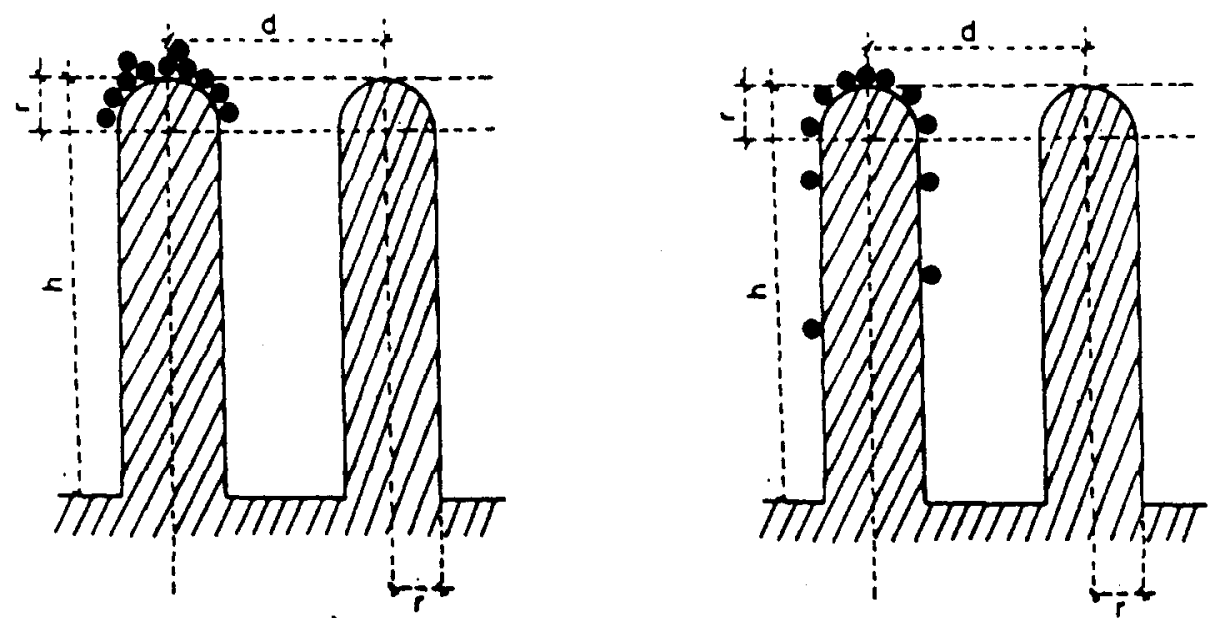

Fig. 13. Particle deposition on a columnar structure. (a) Negligible and (b) non-negligible surface diffusion of particles on their own 2-D domains. 


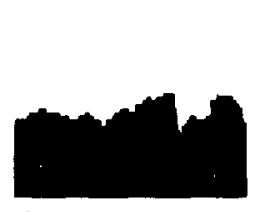

a

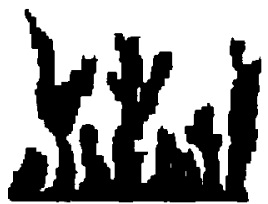

$c$

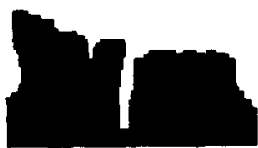

d

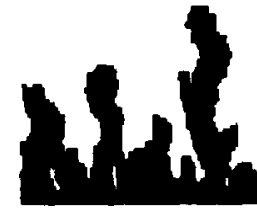

b

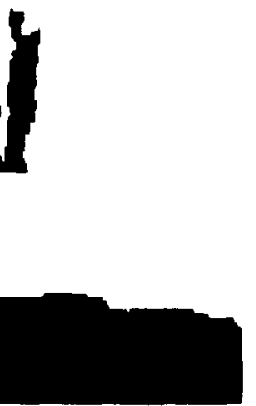

e

Fig. 14. Snapshots obtained from Monte Carlo simulations of the nucleation and growth model. Snapshots (a)-(c) are obtained taking $n=1000, n^{*}=2, l_{\mathrm{a}}=3$ and (a) $P_{n}=0.01$, (b) $P_{n}=0.45$, (c) $P_{n}=1.0$. Snapshots (d) and (c) are obtained by using $n=1000, n^{*}=2, P_{n}=1.0$ and (d) $l=7$,

(e) $l_{\mathrm{a}}=11$ (reproduced with permission from Ref. [39]).

number of particles in the initial ideally flat 2-D substrate profile. The value of $R$ increases as $P_{\eta}$ is changed from 0.01 to 1 ; so the 2-D surface profile can be changed from that of a stepped profile, to that of a columnar profile, and finally to that of a branched columnar 2-D profile. The effect of surface diffusion on the grown patterns has been simulated by changing $l_{\mathrm{a}}$. Thus, for $n=1000, n^{*}=2$ and $P_{n}=$ 1 , the snapshots show a net decrease of $R$ as $l_{n}$ is increased from 1 to 12 . The 2-D profile changes from the branched columnar pattern (Fig. 14c) to the stepped 2-D profile (Fig. 14d, e). As should be expected, surface atom diffusion promotes smoothing of the growing profiles leading to compact structures.

The Monte Carlo simulations based upon this nucleation and growth model, yield $R \propto n$ and $R \propto$ $P_{\eta}^{1 / 2}$ (Fig. 15a and b) relationships, in agreement with the $R \propto q$ and $R \propto j_{\mathrm{M}}^{1 / 2}$ relationships observed for metal electrodeposits (Figs 6 and 7). The proposed model therefore captures the essential features of roughness development at surfaces of real electrodeposits.

The present results can be interpreted considering that $R$ depends on both the deposited mass and the particle mobility, as expressed by [39]:

$$
R \propto n / l_{1}+l_{\mathbf{a}}
$$

and

$$
l_{\mathrm{i}}=D_{\mathrm{i}} / P_{\eta}^{1 / 2},
$$

where $l_{i}$ is the maximum diffusion length of the particles before sticking, and $D_{i}$ is the diffusion coefficient of the particles in the depositing phase. The model implies two basic contributions competing for the roughness development, namely the accumulation of particles at the growing phase and the surface

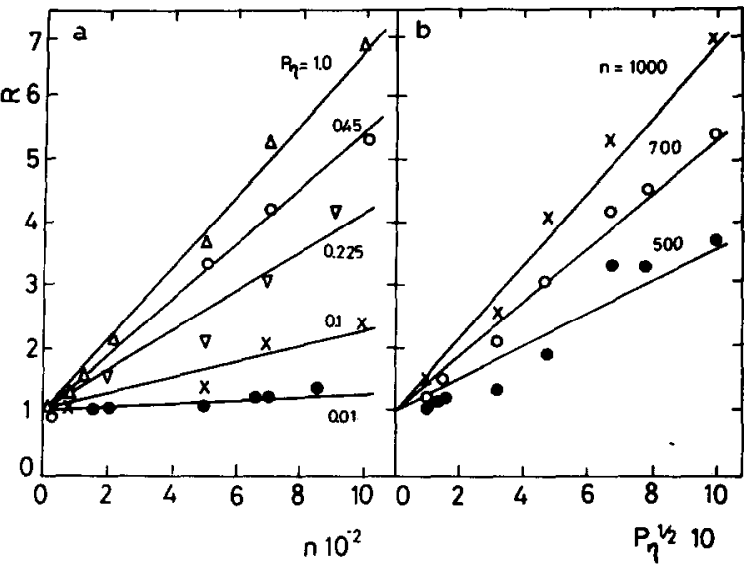

Fig. 15. (a) $R$ vs. $n$ plot for different $P_{\eta}$ values. (b) $R$ vs. $P_{\eta}^{1 / 2}$ for different $n$ values (reproduced with permission from Ref. [39])

mobility of metal atoms leading to surface smoothing.

Monte Carlo simulations were also applied to study the effect of foreign adsorbates on the roughness development. During the growth of the deposit it was found that foreign particle adsorption at low coordination sites produces a decrease in roughness (Fig. 16)[62]. This fact explains the marked decrease

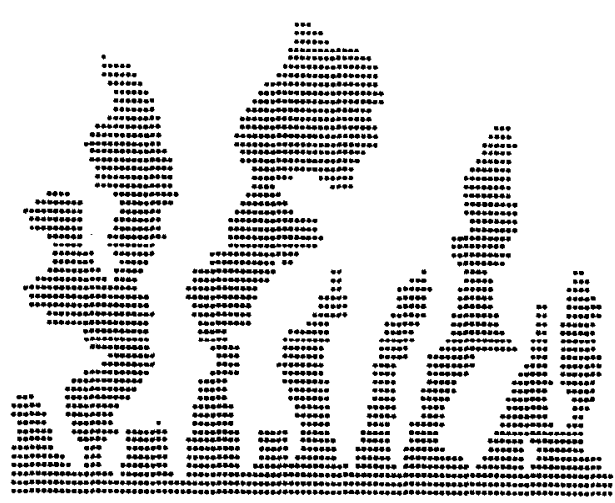

a

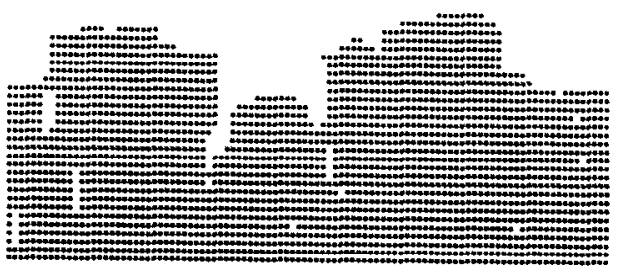

b

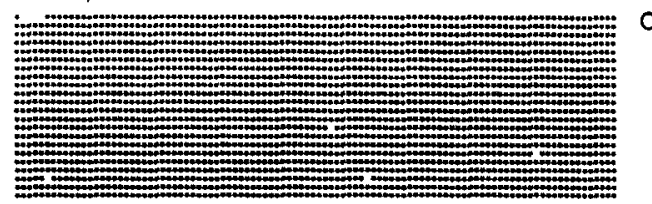

Fig. 16. Snapshots of deposits generated using $P_{1}=1$, $n^{*}=2, n=2200, l_{2}=4$ for different sticking probabilities at low coordination sites, $P_{\mathrm{e}} ;$ (a) $P_{\mathrm{c}}=0.3$, (b) $P_{\mathrm{e}}=0.1$, (c) $P_{e}=0.01$ (reproduced with permission from Ref. [62]). 


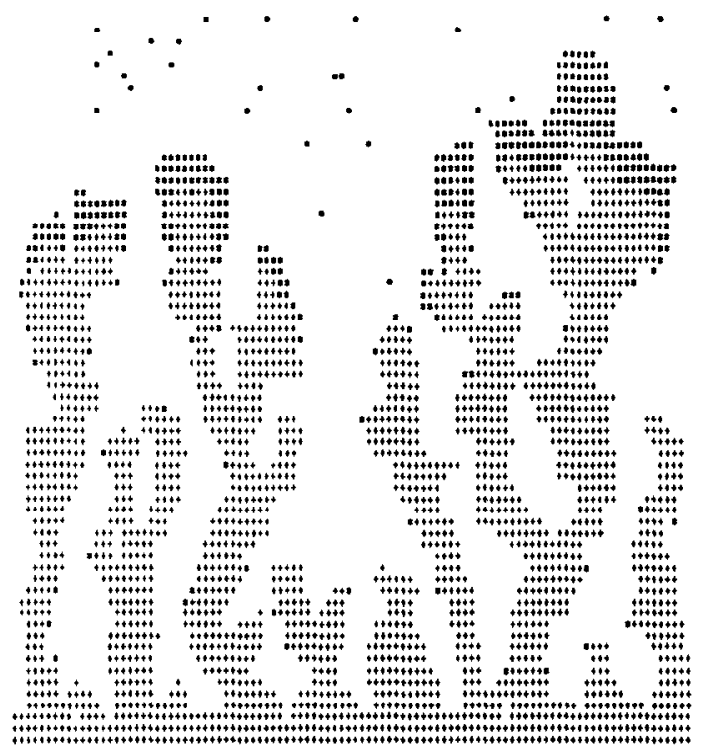

Fig. 17. Snapshot for the nucleation and growth of a deposit on a rough columnar substrate. (+) substrate; $\left({ }^{*}\right)$ deposited particles; $(O)$ particles in the "diluted" solution. The diffusion length, $l_{a}$, is equal to 3 (reproduced with permission from Ref. [61]).

in $R$ observed when $\mathrm{Pt}$ electrodeposits are grown from the electroreduction of Pt oxides in the potential range where the $\mathrm{H}$ atom adsorption takes place[62].

Reactions on rough electrodes have also been studied by Monte Carlo simulations[63]. The snapshots of the nucleation and growth of a deposit at $P_{\eta}=1$ from a "diluted" solution to simulate mass transport control on a rough columnar substrate, reveals a clear shadowing effect (Fig. 17)[61]. In this case, in contrast to tip sites, the inner part of the substrate structure becomes practically inaccessible to the depositing particles. If the diffusion length of the depositing particles is increased on 2-D domains at tip sites, then 3-D metal deposition becomes practically hindered, so the Monte Carlo simulation explains the simple scheme already shown in Fig. 12.

\section{A FRACTAL APPROACH TO ROUGH ELECTRODE SURFACES}

The Monte Carlo simulation discussed in the preceding section allows the conclusion that weakly disordered surfaces with profiles comparable with that depicted in Fig. 4b can be generated through growth processes controlled by either the diffusion of surface atoms or the transport of ions from the bulk of the solution with a non-negligible contribution of surface atom diffusion (Fig. 14a, $d$ and e). On the other hand, a mass transport controlled growth with negligible surface diffusion generates columnar structures with a strong surface disorder (Fig. 14b and c). The latter display can, in principle, be identified with that shown in Fig. 4c. Likewise, the columnar structures resemble those patterns that can be generated through either a diffusion limited deposition grown in the limit of high anisotropy[63] or ballistic depo- sition models[64] which are known to be surface fractals. This similarity of the growing patterns suggests that the rough metal surfaces grown far from equilibrium conditions can be also described as fractals[29]. This assumption is supported by the STM-SEM imaging of those surfaces which exhibit self-similar void patterns covering the nanometre to micrometre range[65]. In this case $D_{\mathrm{s}}$, the fractal dimension of the deposit surface, provides relevant information about the geometry[29], the growth mechanism[66] and the chemical reactivity [9] of these systems.

\subsection{Fractal measurements of surfaces applicable to electrochemistry}

The demonstration of fractality of real systems is a rather complex problem as the fractal behaviour of the object can be constrained to certain length scales (between inner and outer cut-offs). Likewise, the proper experimental and data processing methods for the determination of the fractal dimension cover only a limited range of length scales.

Several experimental methods have been proposed for the evaluation of $D_{s}[66]$, and some of these methods have already been successfully applied to a number of electrochemical systems. One of these methods is concerned with the determination of $M$, the mass, and $R_{z}$, the radius of gyration of the object. $M$ and $R_{z}$ are related to the fractal dimension through the following expression[66]:

$$
M \propto R_{z}^{D_{\mathbf{H}}} .
$$

$M$ and $r$ can be evaluated from digitized images of the evolving object. For this purpose high quality images are necessary as poor contrast makes digitization rather difficult. A modification of this method was used to obtain the fractal dimension of 3-D Cu electrodeposits grown under diffusion control at the tip of a thin $\mathrm{Cu}$ wire[67]. In this case, at each stage of the growing electrodeposit the value of $\boldsymbol{R}_{2}$ was determined from the diffusion-controlled $\mathrm{Cu}$ electrodeposition current at constant potential, and the value of $M$ was derived from the corresponding transferred charge.

An effective fractal dimension of deposits nucleating on a $d_{\mathrm{s}}$ dimensional surface can be obtained from[66]:

$$
M \propto h^{D_{s}-d_{s}},
$$

where $h$ is some measure of the deposit thickness. The fractal dimension of $Z n$ electrodeposits grown on a linear cathode as essentially a 2-D deposit[68] was derived from Equation (15).

Another possibility for determining $D_{s}$ is through the perimeter $(P)$ vs. area $(A)$ relationships of lake or island patterns generated by the intersection of a plane with the rough metal surface. It is known that the intersection of a plane with a self-similar or a self-affine surface generates self-similar lakes or islands. For the latter, the following relationship holds[29]:

$$
P \propto A^{D_{\mathrm{p}} / 2},
$$

where $D_{\mathrm{p}}$ is the fractal dimension of either lakes or islands. Accordingly, $D_{s}$ can be estimated from $D_{p}$, the fractal dimension of the perimeter, through the 
following Equation[29]:

$$
D_{\mathrm{s}}=D_{\mathrm{p}}+1 \text {. }
$$

In this way, SEM images have been used to characterize fractal surfaces of solid materials at the nanometre level[69]. However, SEM microscopy requires 3-D image reconstruction as it gives only 2-D patterns. The perimeter-area method for fractal dimension determination based on STM imaging has been proposed recently[70]. This procedure was presented at this Symposium[43] in relation to the fractal dimension of vapour deposited and electrodeposited Au layers.

Fourier analysis of STM data has also been employed to characterize the surface of solid materials at the nanometre level[71]. In this method every line of the image is transformed with a Fourier routine, so that the transforms are averaged and the power spectrum is calculated. Then, the power spectra are integrated from high frequency and the slope determined by linear regression. The value of $\beta^{\prime}$, the slope of the linear part of the spectrum in a $\log -\log$ plot, is related to $D_{3}$ through the equation:

$$
D_{\mathrm{s}}=2.0+\beta^{\prime} / 2
$$

The value of $D_{\mathrm{s}}$ of rough solid electrodes can also be determined from the potentiostatic current $(i)$ vs. time $(t)$ relationships used for testing a diffusioncontrolled electrochemical reaction involving soluble species as the test reaction [72]. The method is based upon the Cottrell equation for a fractal electrode surface[72]. Accordingly,

$$
i \propto t^{-\alpha}
$$

and

$$
D_{\mathrm{s}}=2 \alpha+1 \text {. }
$$

Values of $D_{\mathrm{s}}$ for rough Au and Pt electrodeposits[73, 74] derived through this procedure are in agreement with those measured by other methods.

The fractal dimension of rough metal electrode surfaces can also be evaluated from the area $(A)$ vs. volume $(V)$ relationships. In this case, the functional- ity between $A$ and $V[29]$ is:

$$
A \propto V^{D_{\mathbf{V}} / 3} \text {. }
$$

This method has been applied to determine the value of $D_{1}$ for dendritic $\mathrm{Ag}$ surfaces[32]. In this case, the value of $A$ is obtained from the upd voltammogram of a foreign metal $(\mathrm{Pb}, \mathrm{Cd})$ used as a yardstick, and the value of $V$ is measured from the electrodeposited charge obtained at a constant potential.

Finally, in the last few years impedance measurements have also been proposed for this purpose[75, 76]. This procedure, however, is still under discussion because no clearcut correlation between $n$, the frequency dispersion exponent of impedance data, and $D_{8}$ has been obtained so far[74].

\subsection{Fractal dimension: characterization, growth mechanisms and reactivity of rough surfaces}

Table 1 summarizes the $D_{8}$ values obtained in our laboratories by using various methods for 3-D metal electrodeposits grown under different experimental conditions. For comparison, data obtained for vapour deposited metal layers and smooth metals are also included.

In our case all electrodeposits prepared at high growth rates far from the equilibrium conditions exhibit $D_{\mathrm{s}}=2.5$. Otherwise, for a nearly constant roughness relaxation time in the electrolyte solution at open circuit, the $D$, value for $A u$ decreases from 2.5 to $2.3-2.2$, in contrast to the $D_{\mathrm{s}}$ value for $\mathrm{Pt}$ which remains practically unchanged. This difference in the surface relaxation rates between $\mathrm{Au}$ and $\mathrm{Pt}$ is directly related to the differences in the surface mobilities of $\mathrm{Au}$ and $\mathrm{Pt}$ atoms at room temperature. On the other hand, for $\mathrm{Au}$ electrodeposits grown at a low rate one obtains $D_{\mathrm{s}}=2.3$.

When the preceding van es of $D_{\mathrm{s}}$ are compared with the $D_{s}$ value resulting for a non-fractal smooth surface, $D_{s}=2$, one can conclude that compact and weakly disordered surfaces are formed under low growth rates or under high growth rates with nonnegligible surface atom diffusion contribution. Otherwise, open structures with strongly disordered

\begin{tabular}{|c|c|c|c|c|}
\hline Metal deposit & Growth rate/nm s ${ }^{-1}$ & Roughness relaxation & $D_{\mathrm{s}} \pm 0.1$ & Method \\
\hline EDAuL & 100 & - & 2.5 & STM \\
\hline EDAuL & 100 & - & 2.5 & $i$ vs. $t$ \\
\hline EDAuL & 100 & $\begin{array}{c}10^{4} \mathrm{~s} ; 325 \mathrm{~K} \\
0.5 \mathrm{M} \mathrm{H}, \mathrm{SO}\end{array}$ & 2.3 & STM \\
\hline EDAuL & 100 & $\begin{array}{l}10^{5} \mathrm{~s} ; 298 \mathrm{~K}^{4} \\
0.5 \mathrm{M} \mathrm{H}_{2} \mathrm{SO}_{4}\end{array}$ & 2.3 & $i$ vs. $t$ \\
\hline Au (smooth) & - & - & 2.0 & $i$ vs. $t$ \\
\hline EDÁuL & 0.1 & - & 2.3 & STM \\
\hline EDPtL & 5 & - & 2.5 & STM \\
\hline EDPtL & 5 & $\begin{array}{c}6 \times 10^{4} \mathrm{~s} ; 325 \mathrm{~K} \\
0.5 \mathrm{M} \mathrm{H}_{2} \mathrm{SO}_{4}\end{array}$ & 2.5 & STM \\
\hline EDAgL & 6400 & - & 2.5 & $A$ vs. $V$ \\
\hline VPAuL & 30 & - & 2.7 & STM \\
\hline
\end{tabular}
surfaces are produced under high growth rates and negligible surface atom diffusion contribution. This picture agrees with the predictions of Monte Carlo

Table 1. Values of $D_{s}$ obtained for different metals

EDMeL, electrodeposited metal layer; VPMeL, vapour deposited metal layer. 
calculations and it proves that the value of $D_{\mathrm{s}}$ can be taken as a measure of the disorder at the solid surface describing the essential features of rough solid surfaces.

The value of $D_{s}$ also contains information about the growth mechanism of the rough metal overlayer. Thus, vapour deposited Au films grown on glass at a high rate and $298 \mathrm{~K}$ are characterized by $D_{s}=2.7$ (Table 1). This Figure agrees with that obtained from large-scale computer simulations of 3-D deposits generated by ballistic deposition models[66]. In these models particles are incorporated in the deposit following random ballistic (linear) trajectories. When a particle contacts the growing clusters at some point it is stopped and added to the cluster at this position. This procedure generates compact structures with rough self-affine fractal surfaces. This picture agrees very well with the STM data, the $R$ vs. $h$ (Fig. 8) and the $\zeta$ vs. $h$ (Fig. 10) plots of Au vapour deposits.

On the other hand, electrodeposits grown far from equilibrium under a negligible surface diffusion contribution imply $D_{s}=2.5$. This figure indicates that another growth mechanism operates for the metal layer electrodeposition. For dendritic $\mathrm{Ag}$ which has been grown through the electroreduction of $\mathrm{Ag}^{+}$ ions in aqueous solutions, the electrodeposit structure looks like that of a self-similar fractal with $D_{8}=$ 2.5. This figure can be associated with a Laplacian growth where the electric field is screened by the supporting electrolyte. The kinetics of this process has been extensively discussed in this Symposium[77]. It is known that Laplacian growth originates DLA-type patterns[66]. Large-scale computer simulations for 3-D deposits generated by diffusion limited aggregation (DLA) models yield $D_{3}=$ 2.5, a Figure which coincides with that determined for electrodeposited $\mathrm{Ag}$ dendrites[32]. It should be noted that $D_{\mathrm{s}}=2.5$ also characterizes self-similar 3-D $\mathrm{Cu}$ electrodeposits grown from aqueous solutions under diffusion control[67].

It should be borne in mind that the mechanistic conclusions derived from $D_{1}=2.5$ for metal deposits grown from the electroreduction of metal oxides cannot at present be regarded as definite ones. In this case, the surface topography appears as a selfaffine rather than a self-similar one, as should be expected for simple DLA models. However, deposit structures resulting from computer simulations for diffusion limited deposition exhibit a certain degree of anisotropy[66]. Computer simulations and experimental work are in progress in our laboratory to clarify this point.

Finally, there is an influence of the fractal characteristics of rough metal surface electrodes on the different types of electrochemical reactions. Thus, Equations (18) and (19) show the behaviour expected for potentiostatic current transients of a diffusioncontrolled reaction at a fractal electrode surface. In this case, the $i \propto t^{-0.5}$ behaviour is replaced by the more general $i \propto t^{-\alpha}$ relationship, the exponent $\alpha$ being related to $D_{s}$. Similar changes are also expected in the slope of the Tafel relationships for charge transfer controlled reactions. Electrochemical processes involving phase formation on fractal surfaces show, as a final example, marked changes with respect to those occurring on smooth electrode surfaces $[61,78]$.

In conclusion, the fractal characterization of rough electrodes opens new possibilities and deserves further research work for explaining the behaviour of real electrode surfaces in many areas of interfacial electrochemistry.

Acknowledgements-The authors thank CONICET (Argentina) and CICYT (Spain) for financial support.

\section{REFERENCES}

1. K. J. Vetter, Electrochemical Kinetics, p. 539. Academic Press, New York (1967).

2. T. Kessler, A. M. Castro Luna, W. E. Triaca and A. J. Arvia, J. appl. Electrochem. 16, 693 (1986).

3. A. T. Hubbard, Accs Chem. Res. 13, 177 (1980).

4. J. Clavilier, Electrochemical Surface Science (Edited by M. P. Soriaga), Ch. 14, p. 201. The American Chemical Society, Washington, D.C. (1988).

5. G. Somorjai, Chemistry in Two Dimensions: Surfaces. Cornell University Press, Ithaca (1981).

6. A. J. Arvia, Surf. Sci. 181, 78 (1987).

7. A. T. Hubbard, J. L. Stickney, M. P. Soriaga, V. K. Chia, S. D. Rosasco, B. C. Schardt, T. Solomun, D. Song, J. White and A. Wiescowski, J. electroanal. Chem. 168,43 (1984).

8. M. E. Hanson and E. Yeager, Electrochemical Surface Science (Edited by M. P. Soriaga), Ch. 10, p. 141. The American Chemical Society, Washington, D.C. (1988).

9. P. Pfeifer and M. Obert, A Fractal Approach to Heterogeneous Chemistry (Edited by D. Avnir), p. 11. Wiley, Chichester (1989)

10. G. A. Somorjai, J. phys. Chem. 94, 1013 (1990).

11. J. Wiechers, H. Brune, T. Gritsch, T. Hofer and R. J. Behm, Phys. Rev. Lett. 62, 59 (1989).

12. F. Jona and P. Marcus, The Structure of Surfaces II Springer, Berlin (1988).

13. N. Furuya and S. Koide, Surf. Sci. 220, 18 (1989).

14. A. Hamelin, Modern Aspects of Electrochemistry (Edited by B. E. Conway, R. White and J. O'M. Bockris), Vol. 16, p. 1. Plenum Press, New York (1985).

15. D. M. Kolb, Advances in Electrochemistry and Electrochemical Engineering (Edited by $\mathrm{H}$. Gerischer and $\mathrm{C}$. W. Tobias), Vol. 11. Wiley, New York (1978).

16. K. Jüttner and W. J. Lorenz, Z. phys. Chem. N.F. 122 , 163 (1980).

17. N. M. Markovic, A. V. Tripkovic, N. S. Marinkovic and R. R. Adzic, Electrochemical Surface Science (Edited by M. P. Soriaga), Ch. 34, p. 498. The American Chemical Society, Washington, D.C. (1988).

18. E. P. Leiva, E. Santos, R. M. Cerviño, M. C. Giordano and A. J. Arvia, Electrochim. Acta 30, 1111 (1985).

19. B. Love and J. Lipkowski, Electrochemical Surface Science (Edited by M. P. Soriaga), Ch. 33, p. 485. The American Chemical Society, Washington, D.C. (1988).

20. S. Bilmes, M. C. Giordano and A. J. Arvia, J. electroanal. Chem. 225, 183 (1987); Can. J. Chem. 66, 2259 (1988).

21. B. D. Cahan, H. M. Villullas and E. B. Yeager, J. electroanal. Chem. 306, 213 (1991).

22. J. Wiecher, T. Twomey, D. M. Kolb and R. J. Behm, J. electroanal. Chem. 248, 451 (1988).

23. D. J. Trevor, C. E. Chidsey and D. N. Loiacono, Phys. Rev. Lett. 62, 929 (1989).

24. D. Margheritis, R. C. Salvarezza, M. C. Giordano and A. J. Arvia, J. electroanal. Chem. 229, 327 (1987).

25. D. C. Alonzo and B. Scharifker, J. electroanal. Chem. 274, 167 (1989) 
26. A. J. Arvia, J. C. Canullo, E. Custidiano, C. Perdriel and W. E. Triaca, Electrochim. Acta 31, 1359 (1986).

27. L. Vázquez, J. M. Gómez Rodríguez, J. Gómez Herrero, A. M. Baró, N. García, J. C. Canullo and A. J. Arvia, Surf. Sci. 181, 98 (1987).

28. J. Gómez, L. Vázquez, A. M. Baró, N. Garcia, C. Perdriel, W. E. Triaca and A. J. Arvia, Nature 323, 612 (1986).

29. B. B. Mandelbrot, The Fractal Geometry of Nature. Freeman, San Francisco (1977).

30. J. J. Fripiat, A Fractal Approach to Heterogeneous Chemistry (Edited by D. Avnir), p. 331. Wiley, Chichester (1989).

31. S. Trasatti and O. Petrii, Pure appl. Chem. 67, 711 (1991)

32. A. Hernández Creus, P. Carro, S. González, R. C. Salvarezza and A. J. Arvia, J. electrochem. Soc. in press.

33. A. J. Arvia, R. C. Salvarezza and W. E. Triaca, Electrochim. Acta 34, 1057 (1989).

34. A. Chialvo, W. E. Triaca and A. J. Arvia, J. electroanal. Chem. 146, 93 (1983).

35. A. R. Despic and K. I. Popov, Modern Aspects of Electrochemistry (Edited by B. E. Conway and J. O'M Bockris), Vol. 7, p. 199. Butterworths, London (1972)

36. M. Vela, R. C. Salvarezza and A. J. Arvia, Electrochim. Acta 35, 117 (1990).

37. L. Vázquez, A. Bartolomé, A. M. Baró, C. Alonso, R. C. Salvarezza and A. J. Arvia, Surf. Sci. 215, 171 (1989).

38. D. Srolowitz, A. Mazor and B. G. Bukiet, J. Vac. Sci. Technol. A6, 2371 (1988).

39. R. C. Salvarezza, C. Alonso, J. M. Vara and A. J. Arvia, Phys. Rev. B41, 12,502 (1990).

40. P. Carro, A. Hernández Creus, S. González, R. C. Salvarezza and A. J. Arvia, J. electroanal. Chem. 310, 361 (1991).

41. P. Herrasti, P. Ocón, L. Vázquez, R. C. Salvarezza, J. M. Vara and A. J. Arvia, 42 ISE Meeting, Montreaux (1991).

42. J. J. Bikerman, Physical Surfaces. Academic Press, New York (1970).

43. P. Herrasti, P. Ocón, L. Vázquez, R. C. Salvarezza, J. M. Vara and A. J. Arvia, Sth International Fischer Symposium, Karlsruhe (1991).

44. C. Alonso, R. C. Salvarezza, J. M. Vara, A. J. Arvia, L. Vázquez, A. Bartolomé and A. M. Baró, J. electrochem. Soc. 137, 2161 (1990).

45. C. Tang, S. Alexander and R. Bruisma, Phys. Rev. Lett. 64, 772 (1990).

46. R. A. Roy and R. Messier, Mater. Res. Soc. Symp. Proc. 38, 363 (1985).

47. N. A. Gjostein, Surfaces and Interfaces I (Edited by J. Burke, N. Reed and W. Weiss), p. 271. Syracuse University Press, New York (1967).

48. J. González Velasco, personal communication.

49. H. P. Bonzel, Surface Physics of Materials (Edited by J. M. Blakeley), p. 280. Academic Press, New York (1975).

50. C. Alonso, R. C. Salvarezza, J. M. Vara and A. J. Arvia Electrochim. Acta 35, 1331 (1990).
51. R. C. Salvarezza, J. M. Vara and A. J. Arvia, Langmuir, in press.

52. S. Glasstone, K. J. Laidler and H. Eyring, The Theory of Rate Processes. McGraw-Hill, New York (1940).

53. G. Estiú, S. Maluendes, E. Castro and A. J. Arvia, J. phys. Chem. 92, 2512 (1988).

54. G. Estiú, S. Maluendes, E. Castro and A. J. Arvia, J. electroanal. Chem. 282, 303 (1990).

56. A. M. Castro Luna, M. C. Giordano and A. J. Arvia, J. electroanal. Chem. 259, 173 (1989).

57. M. L. Marcos, J. M. Vara, J. González Velasco and A. J. Arvia, J. electroanal. Chem. 224, 189 (1987).

58. B. Beden, C. Lamy, N. R. de Tacconi and A. J. Arvia, Electrochim. Acta 35, 69 (1990).

59. B. Beden, F. Hahn, C. Lamy, J. M. Léger, N. R. de Tacconi, R. O. Lezna and A. J. Arvia, J. electroanal. Chem. 261, 401 (1989)

60. K. Kinoshita and P. Stonehart, Modern Aspects of Electrochemistry (Edited by B. E. Conway and J. O'M Bockris), Vol. 12, p. 183. Plenum Press, New York (1977).

61. M. Martins, R. C. Salvarezza, J. M. Vara and A. J. Arvia, J. electrochem. Soc. 138, 2509 (1991).

62. R. C. Salvarezza, C. Alonso, J. M. Vara and A. J. Arvia J. electrochem. Soc. 138, 2938 (1991).

63. P. Meakin, Phys. Rev. A33, 1984 (1986).

64. P. Meakin, P. Ramanlal, L. M. Sander and R. Ball, Phys. Rev. A34, 5091 (1986).

65. M. Gómez-Mateos, L. Vázquez, R. C. Salvarezza, J. M Vara and A. J. Arvia, J. electroanal. Chem. 317, 125 (1991).

66. T. Vicsek, Fractal Growth Phenomena. World Scientific, London (1989).

67. R. M. Brady and R. C. Ball, Nature 309, 225 (1984).

68. M. Matsushita, Y. Hayakawa and Y. Sawada, Phys. Rev. A32, 3814 (1985).

69. F. Vernier, C. Dussert, G. Rasigni and M. Rasigni, J. Vac. Sci. Technol. A6, 1627 (1988).

70. J. Gómez Rodríguez, A. M. Baró, L. Vázquez, R. C. Salvarezza, J. M. Vara and A. J. Arvia, J. phys. Chem. 96, 347 (1992).

71. M. W. Mitchell and D. A. Bonnell, J. Mater. Res. 5, 2244 (1990).

72. T. Pajkossy and L. Nyikos, Electrochim. Acta 34, 171 (1989).

73. P. Ocón, P. Herrasti, L. Vázquez, R. C. Salvarezza, J. M. Vara and A. J. Arvia, J. electroanal. Chem. in press.

74. T. Pajkossy, J. electroanal. Chem. 300, 1 (1991).

75. A. Le Mehauté and G. Crepy, Solid St. Ionics 9-10, 17 (1983).

76. S. H. Liu, Phys. Rev. Lett. 55, 529 (1985)

77. A. Hernández-Creus, P. Carro, S. González, R. C. Salvarezza and A. J. Arvia, Electrochim. Acta 37, 2215 (1992).

78. M. E. Martins, R. C. Salvarezza and A. J. Arvia, Electrochim. Acta 36, 1617 (1991). 